\title{
Guest editorial: Genetic and epigenetic alterations in hematopoietic malignancies
}

\author{
Toshio Kitamura
}

Received: 21 January 2013/Accepted: 22 January 2013/Published online: 9 February 2013

(C) The Japanese Society of Hematology 2013

It is now recognized that multiple gene alterations are required for the development of leukemia, as is the case with solid tumors. About a decade ago, these gene alterations were classified into two groups, called class I and class II mutations [1]. Class I mutations include activating mutations of tyrosine kinase receptors such as FLT-3 and c-Kit, tyrosine kinases such as JAK2, oncogenes such as Ras as well as inactivating mutations of tumor suppressors such as p53 and NF-1. Class I mutations induce cell proliferation or block apoptosis. Class II mutations, which include inactivating mutations of transcription factors such as AML1/Runx1 and chromosome modifying enzymes such as MLL, block hemopoietic differentiation. Class II mutations are often caused by chromosomal translocations resulting in fusion proteins such as AML1-ETO, PMLRARa and a variety of MLL-fusions. Combinations of class I and II mutations but not either class I or class II mutations alone induced acute leukemia in mouse models. In addition, both class I and class II mutations frequently coexist in patients' leukemic cells. Thus, it was assumed that combinations of class I and class II mutations are required for leukemogenesis. On the other hand, class I mutations alone seem to induce myeloproliferative neoplasm (MPN) while class II mutations are associated with myelodysplastic syndromes (MDS), and additional mutations would lead to leukemic transformation of MPN and MDS (Fig. 1). However, recently developed high-speed sequencing and SNP arrays have revealed a variety of novel mutations in hematologic malignancies. Those

\section{T. Kitamura $(\bowtie)$}

Division of Cellular Therapy/Division of Stem Cell Signaling,

The Institute of Medical Science, The University of Tokyo,

Tokyo, Japan

e-mail: kitamura@ims.u-tokyo.ac.jp include mutations of epigenetic regulators, signaling adaptors and proteins involved in splicing [2]. Among these mutations, those in adaptors such as c-Cbl and Lnk may be categorized into class I mutations because mutations of these adaptors with negative signaling roles would lead to autonomous signaling of cytokine receptors. In addition, one of epigenetic regulators mutations, MLLfusions, was categorized with class II mutations. Thus, the "class I and class II story" need to be modified.

In this series of Progress in Hematology, four articles review genetic and epigenetic alterations in hematological malignancies. Drs. Tomoki Naoe and Hitoshi Kiyoi briefly summarize the class I and class II story, and then offer an overview of newly identified mutations of epigenetic regulators. They attempt to integrate these mutations, which they call class III, into the class I and class II story. They also discuss driver and passenger mutations as well as background mutations randomly acquired in hematopoietic cells with age and stress [3]. When we think about epigenetic aspects of diseases, MDS is the most prominent because DNA hypomethylating agents are found to be effective in a substantial numbers of patients with MDS. Drs. Jumpei Yamazaki and Jean-Pierre Issa review the basics of epigenetics in cancer and MDS as well as the therapeutic use of DNA hypomethylating agents in MDS and AML. This review update the history as well as the current state of MDS therapy, including a combination therapy of DNA hypomethylating agents and histone deacetylating enzyme (HDAC) inhibitors.

Concerning MPN, a class I mutation alone such as Bcr$\mathrm{Abl}$ and JAK2V617F (activating mutant of JAK2) induces MPN-like diseases in mouse models, and most patients with MPN harbor either Bcr-Abl or JAK2-V617F. Therefore, it was assumed that these mutations are the sole mutations related to MPN and were able to induce MPN by 


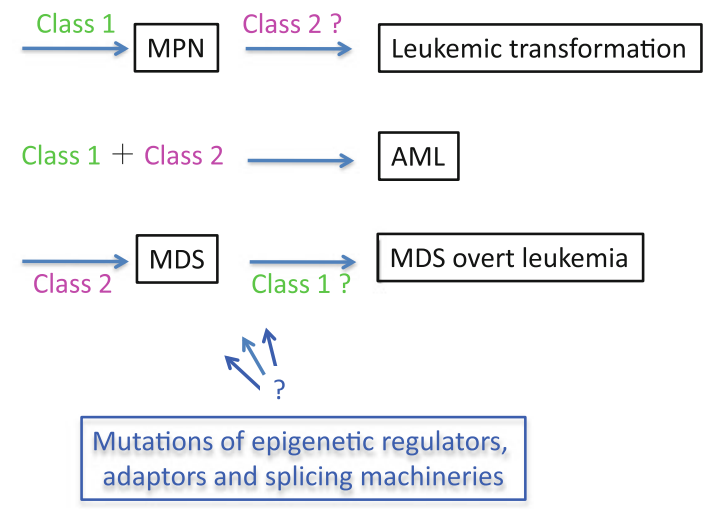

Fig. 1 Combinations of mutations in hematological malignancies. Class 1 class 1 mutations, class 2 class 2 mutations

themselves. However, it has recently been shown that MPN patients also harbor a variety of mutations in epigenetic regulators as well as adaptor proteins. After briefly introducing MPN classifications, Drs. Jelena Milosevic and Robert Kralovics survey the genetic and epigenetic alterations of MPN, including JAK2, thrombopoietin receptor MPL, adaptor proteins LNK and CBL, epigenetic regulators, and proteins involved in the splicing machinery. They focus on the relationship of such somatic mutations and the progression or leukemic transformation of MPN. Dr. Stephen Nimer and his colleagues summarize post-translational protein-modifying enzymes and their relation to leukemogenesis. These enzymes include lysine and arginine methylatransferases, histone acetyltransferases, histone deacethylases, and kinases that phosphorylate serine/ threonine or tyrosine residues of proteins including histones. Among them, they stress the importance of protein arginine methyltransferases (PRMT) in leukemogenesis. In addition to histones, PRMTs methylate a variety of nonhistone proteins including p53. The tumor suppressor p53 can be arginine methylated by PRMT5, which leads to p53dependent G1 arrest. PRMT is a new subject that has recently attracted the attention of the field.
These four reviews present excellent summaries of the mutations found in a variety of proteins, including those involved in the epigenetic process and in the splicing in various hematologic malignancies. However, further work will be required to clarify the pathological meanings of these mutations in malignant transformation. Recent progress in high-speed sequencing frequently identified more than 10 mutations in a single sample of AML, MDS, or MPN. With such data becoming available now, one obvious question is how many mutations or which combinations are required for cell transformation (driver mutations). Personally, I expect that only a couple of mutations will be shown to be required for leukemogenesis and that the other mutations play roles in the progression or modification of the disease, or are irrelevant to the disease. In a mouse leukemia model, combination of a C/EBP $\alpha$ mutation and an activating mutant of tyrosine kinase receptor FLT3 induced acute leukemia in just 2 weeks in all mice [4], suggesting that, at least in some combinations, two mutations would be enough to induce leukemia. I hope that the knowledge summarized in this PIH will help us further advance our understanding of the causative roles of various mutations in the malignant transformation of hematopoietic cells.

\section{References}

1. Gilliland DG. Hematopoietic malignancies. Curr Opin Hematol. 2001;8:189-91.

2. Shih AH, Abdel-Wahab O, Patel JP, Levine RL. The role of mutations in epigenetic regulators in myeloid malignancies. Nat Rev Cancer. 2012;12:599-612.

3. Welch JS, Ley TJ, Link DC, Miller CA, Larson DE, Koboldt DC, et al. The origin and evolution of mutations in acute myeloid leukemia. Cell. 2012;150:264-78.

4. Kato N, Kitaura J, Doki N, Komeno Y, Watanabe-Okochi N, Togami K, et al. Two types of C/EBPa mutations play distinct but collaborative roles in leukemogenesis: lessons from clinical data and BMT models. Blood. 2011;117:221-33. 